\title{
Gene silencing of indoleamine 2,3-dioxygenase hinders tumor growth through angiogenesis inhibition
}

\author{
JINFENG PAN $^{1,2^{*}}$, KENG YUAN $^{1,2^{*}}$, SHANSHAN PENG ${ }^{1,2}$, YANQIN HUANG $^{1,2}$, \\ YUJUAN ZHANG ${ }^{1,2}$, YINYING HU ${ }^{1,2}$, YUANYUAN FENG ${ }^{1,2}$, YANMEI SHI ${ }^{1,2}$, \\ YANLING LIU ${ }^{1,2,4}$, HONGMEI WANG ${ }^{1,2}$, NANJIN ZHOU ${ }^{1,2}$ and WEIPING MIN ${ }^{1,2,3}$ \\ ${ }^{1}$ College of Pharmacy and Institute of Immunotherapy of Nanchang University, and Jiangxi Academy of \\ Medical Sciences; ${ }^{2}$ Jiangxi Provincial Key Laboratory of Immunotherapy, Nanchang, Jiangxi, P.R. China; \\ ${ }^{3}$ Department of Surgery, Pathology, and Oncology, University of Western Ontario, London, \\ Ontario, Canada; ${ }^{4}$ Jiangxi University of Technology, Nanchang, Jiangxi, P.R. China
}

Received December 31, 2016; Accepted March 3, 2017

DOI: 10.3892/ijo.2017.3975

\begin{abstract}
The significance of indoleamine 2,3-dioxygenase-1 (IDO1) has been studied in various types of tumors, but the relationship between IDO1 and tumor angiogenesis needs further delineation. We aimed to clarify the relationship between tumor angiogenesis and IDO1 expression, and to explore the possibility of IDO1-targeting molecular therapy for lung cancer. For the first time, we found that silencing the IDO1 gene using small interfering RNA (siRNA) inhibits in vitro cancer cell invasion and migration. We further demonstrated that knockdown of IDO1 decreased the formation of vasculogenic mimicry. In addition to these in vitro findings, we also demonstrated that in vivo IDO1 gene silencing using short hairpin RNA (shRNA) delayed tumor onset and inhibited tumor growth in the mouse model. Immunostaining showed that IDO1 gene silencing inhibited tumor angiogenesis. Moreover, the expression of IDO1 was associated with microvessel density (MVD) labeled by CD34 and CD146. These findings indicate that IDO1 has the potential to participate in or contribute to the formation of new capillaries, supporting the applicability of IDO1-targeting molecular therapy in lung cancer.
\end{abstract}

Correspondence to: Professor Weiping Min, Department of Surgery, Pathology, and Oncology, University of Western Ontario, London, Ontario N6A 5A5, Canada

E-mail: weiping.min@uwo.ca

${ }^{*}$ Contributed equally

Abbreviations: IDO1, indoleamine 2,3-dioxygenase-1; siRNA, small interfering RNA; shRNA, short hairpin RNA; LLC, Lewis lung cancer; MVD, microvessel density; VM, vasculogenic mimicry; $\mathrm{H} \& \mathrm{E}$, hematoxylin and eosin

Key words: indoleamine 2,3-dioxygenase-1, lung cancer, angiogenesis, siRNA

\section{Introduction}

Lung cancer is an international health problem and its incidence and mortality rate show a rising trend among various common malignant tumors (1). As early lung cancer is often asymptomatic, more than $20 \%$ of non-small cell lung cancers are classified as stage III disease at the time of diagnosis (2). Standard treatment for advanced lung cancer includes surgery and combination chemotherapy, but there are issues with relapse and chemotherapy drug resistance, while the toxicity of chemotherapy also limits its range in clinical applications. Therefore, exploration of new treatments for lung cancer is a hotspot of current research.

Indoleamine 2,3-dioxygenase-1 (IDO1) is a rate-limiting enzyme that catalyzes the catabolism of tryptophan by the kynurenine pathway (3), and its activity has been detected in various tissues such as lung, small intestine and placenta (4), especially in the thymic medulla and secondary lymphoid organs of the T-cell area immune system $(5,6)$. IDO1 expression in tumor tissues is significantly higher than that in normal tissues. However, overexpression of IDO1 gene contributes to the depletion of local tryptophan, resulting in the suppression of T-cell and NK-cell functions. These immune cells are sensitive to tryptophan deficiency. At the same time, the accumulation of tryptophan metabolites, especially kynurenic acid, can inhibit NK cell receptor expression, thereby inhibiting the function of NK cells, thus developing tumor immune tolerance (7). Many studies have shown IDO1 expression in various cancers, including lung (8), colorectal (9), prostate (10), breast (11), and ovarian (12), and the expression of IDO is associated with poor patient prognosis (13-15).

Angiogenesis is a necessary condition for the growth and metastasis of solid tumors. It provides the necessary nutrients for growth, allowing the tumor to enter the rapid growth phase, and provides the channel for distant metastasis of the tumor (16). Microvessel density (MVD) is one of the most important parameters in the evaluation of tumor angiogenesis. As an indirect measure of angiogenesis, MVD can be used to reflect the biological behavior of malignant tumor. MVD has been shown to be an independent prognostic marker to predict 
tumor growth, metastasis and recurrence in many solid tumors including lung cancer (17). CD34 is a highly glycosylated transmembrane protein that allows for quantitative assessment of microvessel density in the event of vascular injury (18). CD34 is one of the markers of vascular endothelium, which is considered to be a repeatable, stable and mature vascular endothelial marker. CD146 is a single-chain membrane through glycoprotein, belonging to the immunoglobulin superfamily members. It has homology with many cell adhesion molecules. CD146 is involved in tumor angiogenesis, not only as a cell adhesion molecule, but also as a membrane signal transduction molecule in tumor-induced angiogenesis (19). We used CD34 and CD146 as markers for quantitative evaluation of microvessel density in this study.

Current research of IDO1 is focused on its immunological function with very few studies on the relationship between IDO1 expression and angiogenesis. In this study, we aimed to expound the relationship between IDO1 expression and lung cancer progression, particularly angiogenesis, and to develop IDO1-targeted molecular therapy to inhibit angiogenesis of lung cancer.

\section{Materials and methods}

Cell lines and experimental animals. The Lewis lung cancer (LLC) cell line used in this study was obtained from the American Type Culture Collection (ATCC) and maintained in DMEM medium (Invitrogen Life Technologies, Carlsbad, CA, USA) with $10 \%$ FBS, L-glutamine, penicillin, and streptomycin at $37^{\circ} \mathrm{C}$ in $5 \% \mathrm{CO}_{2}$. Four- to six-week-old female C57BL/6 mice were purchased from The Jackson Laboratory (Bar Harbour, ME, USA). All experimental protocols and ethics approval were in accordance with the approved guidelines for safety requirements of Jiangxi Academy of Medical Sciences, Nanchang University.

siRNA synthesis and transfection. The siRNA targeting IDO1 mRNA was generated in accordance with the target sequence selection method described by Jin et al (20). siRNA was synthesized by the manufacturer (Sigma, St. Louis, MO, USA). siRNA targeting luciferase gene GL2 (GL2 siRNA) was used as a scrambled-silencing control as GL2 is not expressed in treated cells. IDO1 siRNA and GL2 siRNA were transfected into LLC cells using Lipofectamine-2000 reagent (Invitrogen Life Technologies). Briefly, cells were plated into 12-well plates $\left(1 \times 10^{5}\right.$ cells/well) and allowed to grow overnight to reach approximately $70 \%$ confluence. Cell medium was replaced with $300 \mu 1$ OptiMEM $^{\circledR}$ serum-reduced medium (Invitrogen Life Technologies) before transfection. IDO1 siRNA $(1 \mu \mathrm{g})$ or GL2 siRNA was incubated with $2 \mu \mathrm{l}$ of Lipofectamine-2000 reagent in $200 \mu 1$ of OptiMEM serum-reduced medium at room temperature for $20 \mathrm{~min}$, and then the mixture was gently added to each group.

IDO1 mRNA quantification. Cells were lysed using TRIzol Reagent (Invitrogen Life Technologies) and total RNA was isolated according to the manufacturer's instructions. Total RNA $(1 \mu \mathrm{g})$ was synthesized to cDNA using reverse transcriptase (MMLV-RT, Invitrogen Life Technologies). The following primers sets were used for qPCR amplifications:
Actin, 5'-AGG GAA ATC GTG CGT GAC AT-3' (sense) and 5'-AAC CGC TCG TTG CCA ATA GT-3' (antisense); IDO1, 5'-GTACATCACCATGGCGTATG-3' (sense) and 5'-CGAGGAAGAAGCCCTTGTC-3' (antisense); real-time PCR reactions were performed in a Stratagene Mx3000P QPCR System (Agilent Technologies, Santa Clara, CA, USA) using SYBR green PCR Master Mix (Invitrogen Life Technologies) according to manufacturer's protocol. The differences of gene expression were calculated using the $\Delta \mathrm{Ct}$ method.

Western blot analysis. Cells $\left(1 \times 10^{5}\right)$ were seeded into a 12 -well plate and grown overnight. The cells were transfected with IDO1 siRNA or GL2 siRNA for $48 \mathrm{~h}$. Cells were harvested, washed twice with ice-cold PBS, re-suspended in protein lysis buffer with complete protein inhibitor, and then the container was kept on ice for $30 \mathrm{~min}$. Lysed cells were centrifuged at $12,000 \mathrm{rpm}$ for $10 \mathrm{~min}$ at $4^{\circ} \mathrm{C}$, and the supernatant was collected and stored at $-20^{\circ} \mathrm{C}$ for future use. Protein concentration was determined by Bio-Rad protein assay and $40 \mu \mathrm{g}$ of each group cell lysate was separated on $10 \%$ SDS-PAGE, transferred to nitrocellulose membrane, blocked with $5 \%$ fat-free milk and $3 \%$ BSA in TBST $(0.25 \%$ Tween-20), probed with a mouse anti-human IDO1 mAb (Santa Cruz Biotechnology, Santa Cruz, CA, USA) that binds to both human and mouse IDO1 and monoclonal anti- $\beta$-actin Ab (Santa Cruz Biotechnology) according to the manufacturer's instructions, and visualized by an ECL assay (Pierce, Rockford, IL, USA). The gray scale was calculated by ImageJ software, and the relative expression of IDO1 protein was respectively calculated by IDO1/ $\beta$-actin.

Transwell cell migration and invasion assays. Transwell chambers with an $8 \mu \mathrm{m}$ pore size were used to detect migration and invasion abilities of LLC cells after being transfected with IDO1 siRNA or GL2 siRNA for $24 \mathrm{~h}$. For migration assays, $8 \times 10^{4}$ cells in $200 \mu 1$ DMEM containing $10 \%$ FBS were seeded into the upper chamber and $500 \mu 1$ DMEM containing $20 \%$ FBS was added to the lower chamber. For invasion assays, the upper chamber (BectonDickinson, Bedford, MA, USA) was precoated with $80 \mu 15 \%$ Matrigel overnight before $4 \times 10^{4}$ cells were seeded. Cells were cultured in $5 \% \mathrm{CO}_{2}$ at $37^{\circ} \mathrm{C}$ for $24 \mathrm{~h}$. Cells on the upper side of Transwell were then removed, the wells were fixed with paraformaldehyde for $15 \mathrm{~min}$, and hematoxylin and eosin (H\&E) was used to stain the cells. The migrated and invaded cells were quantified by light microscopy and the number of cells in five high-power fields was counted to represent the migrated cell number.

shRNA expression vector treatment. A lung cancer model was established by inoculating $1 \times 10^{6}$ LLC cells subcutaneously into the upper hind leg of C57BL/6 mice. The cancer-bearing mice were treated with $50 \mu \mathrm{g}$ of IDO1 shRNA or scramble shRNA in $1.0 \mathrm{ml}$ PBS by hydrodynamic injection through the tail vein at days 3, 7, 14 and 21. Tumor onset day was defined as the time when tumor diameter reached $5 \mathrm{~mm}$. Tumor volume was measured by caliper every three days when tumors appeared and the tumor volume was calculated using the following formula: tumor volume $=0.5 \mathrm{x}$ (tumor width) $\mathrm{x}$ (tumor length). Tumors were weighed using an electronic balance. 

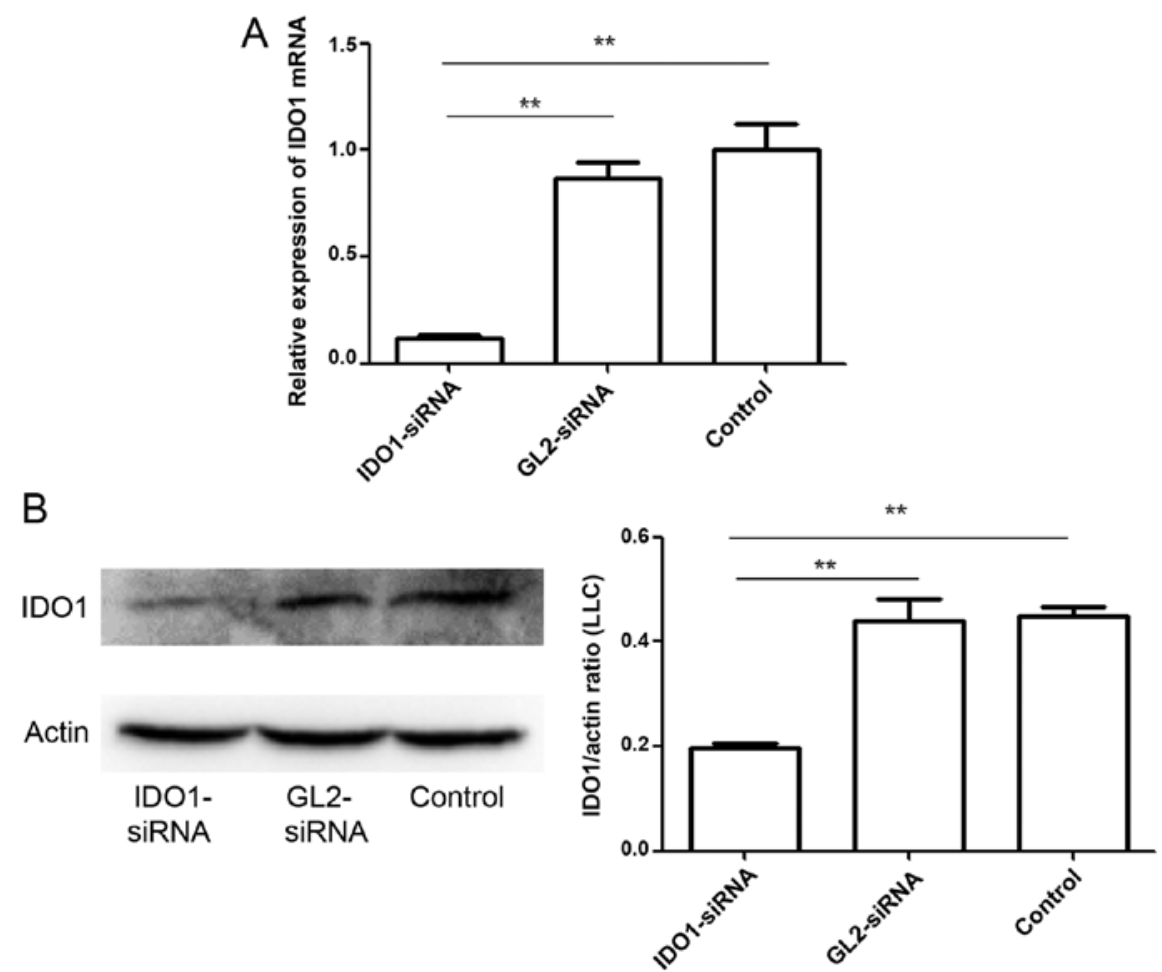

Figure 1. Gene silencing of IDO1 with siRNA in LLC cells. (A) Silencing IDO1 in LLC cell line. LLC cells were transfected with siRNA targeting IDO1 or GL2 (control siRNA), or remained untransfected as a blank control. IDO1 mRNA expression levels were measured $24 \mathrm{~h}$ post-transfection by qRT-PCR as described in Materials and methods. Bars indicate the means of two independent measurements. (B) IDO1 expression at the protein level. LLC cells were transfected with IDO1 siRNA or GL2 siRNA, or remained untransfected as a blank control as described above. After $48 \mathrm{~h}$ transfection, total protein was extracted from cells and separated by PAGE. IDO1 protein expression level in LLC cells was detected by western blotting. Data are expressed as mean \pm SD $(* * 0.01)$.

Vasculogenic mimicry assay in vitro. LLC cells were transfected with siRNA targeting IDO1 or GL2 (control siRNA), or remained untransfected as a blank control. At $24 \mathrm{~h}$ after gene silencing, the $3 \times 10^{4} /$ well cells were placed on the Matrigel. After $3 \mathrm{~h}$ culture, formations of vasculogenic mimicry (VM) were observed under microscope. The numbers of VM tubers formed by the cells transfected with IDO1 siRNA, GL2 siRNA and control cells were counted.

Immunohistochemical staining. Tumor tissues from lung cancer-bearing mice were collected on day 21 and fixed with PFA. Paraffin sections were prepared, deparaffinized, and treated with hydrogen peroxide for 20 min to block endogenous peroxidase. The antigen was repaired with sodium citrate buffer in a pressure cooker. Then, the paraffin sections were reacted with primary antibody for $16 \mathrm{~h}$ at $4^{\circ} \mathrm{C}$ overnight. After 3 washes with PBS, sections were incubated with enzymeconjugated streptavidin for $30 \mathrm{~min}$. The sections were washed with PBS 3 times, and color was developed using the DAB method. The antibodies were as follows: IDO1 (Santa Cruz Biotechnology, 1:50), CD34 (Abcam, Cambridge, MA, USA; 1:250), CD146 (Abcam, 1:250). The degree of immunostaining of sections was reviewed and scored independently by two observers, based on both the proportion of positively stained tumor cells and the intensity of staining. Intensity of staining was recorded on a scale of 0 , no staining; 1 , weak staining; 2 , moderate staining; and 3 , strong staining. The proportion of positively stained tumor cells was graded as follows: 0 , no positive tumor cells; $1,<10 \%$ positive tumor cells; $2,10-50 \%$ positive tumor cells; $3,50-80 \%$ positive tumor cells; and
$4,>80 \%$ positive tumor cells. The staining index $=$ staining intensity x proportion of positively stained tumor cells (21). We evaluated the expression level of IDO1 by staining index (scored as $0,1,2,3,4,6,9$ or 12) using this method. We counted the MVD using the classic method reported by Weidner et al (22). The distribution of blood vessels in the whole slice was observed under light microscopy first, and we selected the most active areas of neovascularization to count microvessels per $200 \mathrm{x}$ field.

Statistical analysis. Numeric data are presented as mean \pm SD. Student's t-test (2-tailed) was used to determine differences between two means. For comparison of multiple groups, one-way ANOVA test was applied. The correlation between MVD and IDO1 protein expression was analyzed by Spearman rank correlation analysis. For all statistical analyses, differences with $\mathrm{p}$-values $<0.05$ were considered significant.

\section{Results}

IDO1 siRNA significantly knocks down IDO1 expression in LLC cells. To test the efficacy of gene silencing using IDO1 siRNA, LLC cells were transfected with IDO1 siRNA or GL2 siRNA (control siRNA). At $24 \mathrm{~h}$ after transfection, IDO1 expression was measured at the transcriptional level by qPCR (Fig. 1A). In the cells with IDO1 siRNA transfection, IDO1 mRNA expression decreased by $>80 \%(\mathrm{p} \leq 0.01)$. IDO1 expression at the protein level was also measured by western blot analysis (Fig. 1B). Data show that the designed IDO1 


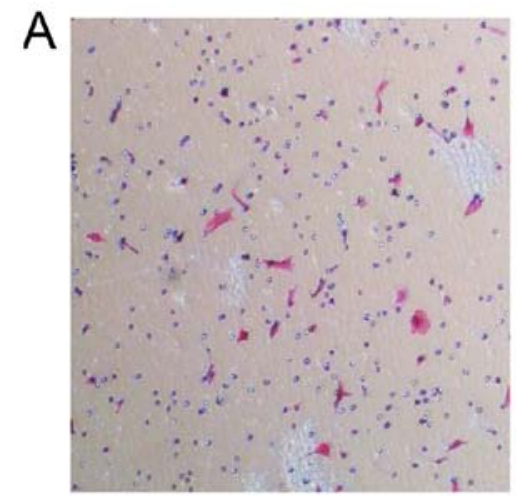

IDO1-siRNA

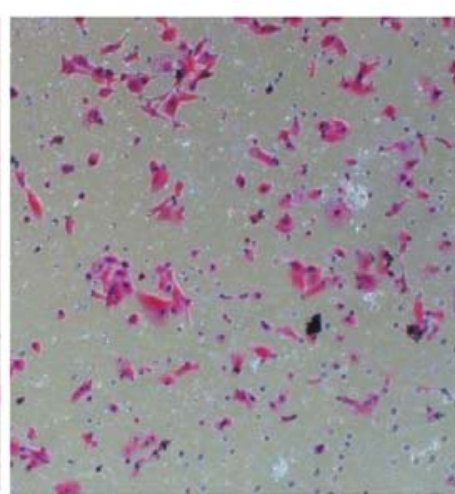

GL2-siRNA

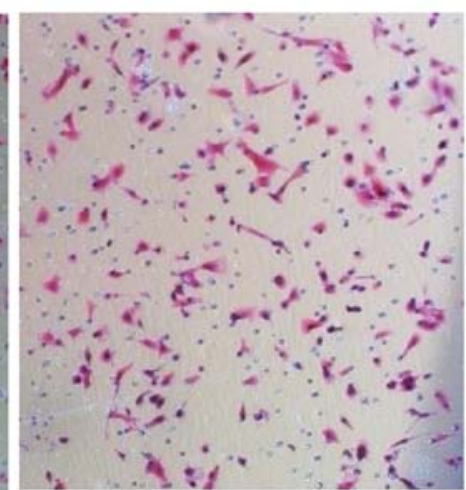

Control

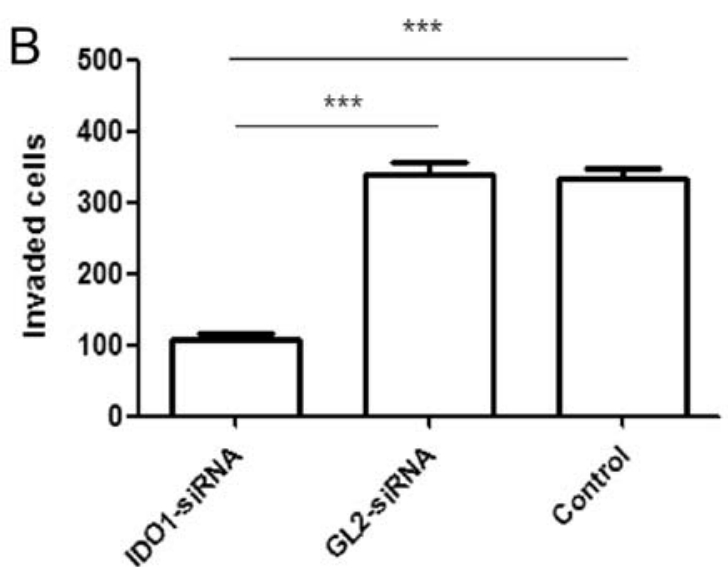

Figure 2. The effect of IDO1 on the invasion ability of LLC cells. (A) LLC cells were transfected with siRNA targeting IDO1 or GL2 (control siRNA), or remained untransfected as a blank control. At $24 \mathrm{~h}$ after gene silencing, the cells were seeded in the 5\% Matrigel of Transwells. The invaded cells were stained with hematoxylin and eosin (H\&E), and observed under microscope. (B) The numbers of invasion cells tranfected with IDO1 siRNA, GL2 siRNA and control cells were counted. Data are expressed as mean $\pm \mathrm{SD}\left({ }^{* * *} \mathrm{p} \leq 0.001\right)$.

siRNA significantly knocked down IDO1 expression in LLC cells.

Effect of IDO1 on the invasion abilities of LLC cells. As shown in Fig. 2, the numbers of invasion cells tranfected with IDO1 siRNA, GL2 siRNA, and control cells were 108.667士6.658/well, $341.333 \pm 16.773 /$ well and $333.333 \pm 16.442 /$ well, respectively, showing significant difference $(\mathrm{p} \leq 0.001)$. In other words, IDO1 expression can decrease the invasive capacity of LLC cells in vitro.

Effect of IDOI on the migration ability of LLC cells. Fig. 3 shows the numbers of migrated cells transfected with IDO1 siRNA, GL2 siRNA, and control cells: 121.333 $\pm 35.921 /$ well, $338 \pm 31.953 /$ well and $380 \pm 61.294 /$ well, respectively, showing a significant difference $(\mathrm{p} \leq 0.001)$. Thus, IDO1 expression can reduce the migration of LLC cells in vitro.

Treatment with IDOI shRNA in vivo suppresses tumor growth. To explore the therapeutic effect of silencing IDO1, we treated tumor-bearing mice with IDO1 shRNA. As shown in Fig. 4A, treatment with IDO1 shRNA delayed tumor onset $(\mathrm{p} \leq 0.01)$. Tumor growth was significantly slower in IDO1 shRNA-treated mice compared with scrambled shRNA-treated mice and control group (Fig. 4B). Both tumor size (Fig. 4C) and weight (Fig. 4D) were less in IDO1
shRNA-treated mice than in scramble shRNA-treated mice and control mice. These results indicate that IDO1 shRNA, injected intravenously, can inhibit tumor growth in a murine lung cancer model.

Effect of IDOI on vasculogenic mimicry formation of LLC cells. Tumor growth and metastasis depend on angiogenesis. Tumor cells, through morphological self-deformation and matrix remodeling, form a unique vascular microcirculation pipeline structure. This channel without endothelial lining is called vasculogenic mimicry (VM) (23). It is an important complement to classical angiogenesis outside the tumor. Fig. 5 shows the numbers of VM tubers formed by the cells transfected with IDO1 siRNA, GL2 siRNA and control cells were $24.67 \pm 4.041 /$ well, $45.67 \pm 3.512 /$ well and $48 \pm 4.583 /$ well, respectively, showing significant difference $(\mathrm{p} \leq 0.001)$. That is, gene silencing of IDO1 can inhibit VM formation.

Effect of IDO1 on tumor angiogenesis. Fig. 6A shows that tumor tissues of mice with scramble shRNA or control group expressed markedly high levels of CD34 and CD146 protein. In experimental tumor tissues from IDO1 shRNA-treated mice, there were significantly decreased levels of CD34 and CD146 protein expression. Fig. 6B shows that the number


newly formed blood vessels in the IDO1 shRNA tumors was 




IDO1-siRNA

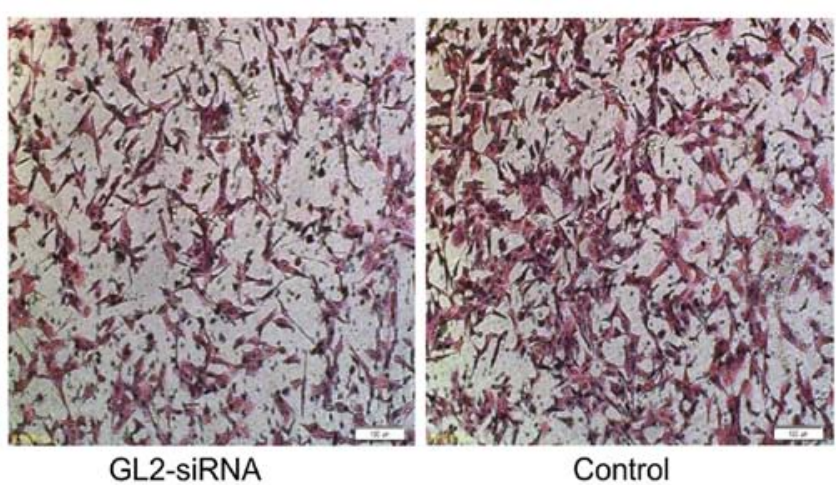

$\star * *$

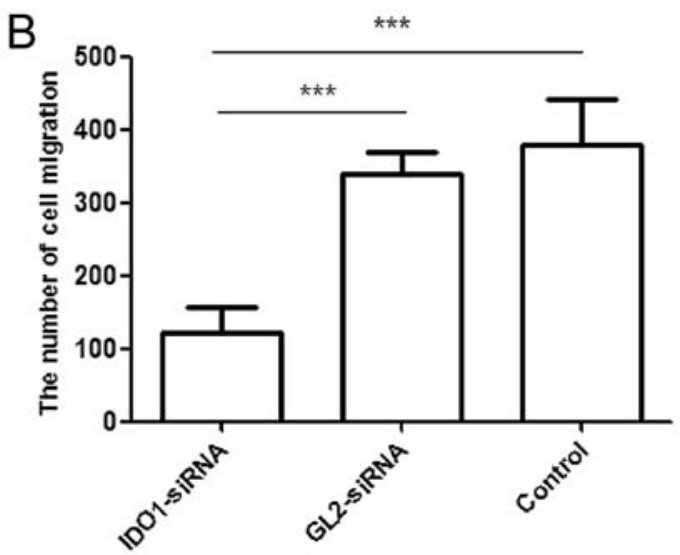

Figure 3. The effect of IDO1 on the migration ability of LLC cells. (A) LLC cells were transfected with siRNA targeting IDO1 or GL2 (control siRNA), or remained untransfected as a blank control. At $24 \mathrm{~h}$ after gene silencing, the cells were seeded in the upper chambers of Transwells. Cells that migrated to the bottom cells were stained with hematoxylin and eosin (H\&E), and observed under a microscope. (B) The number of migrated cells transfected with IDO1 siRNA, GL2 siRNA and control cells was counted. Data are expressed as mean $\pm \mathrm{SD}\left({ }^{* * *} \mathrm{p} \leq 0.001\right)$.
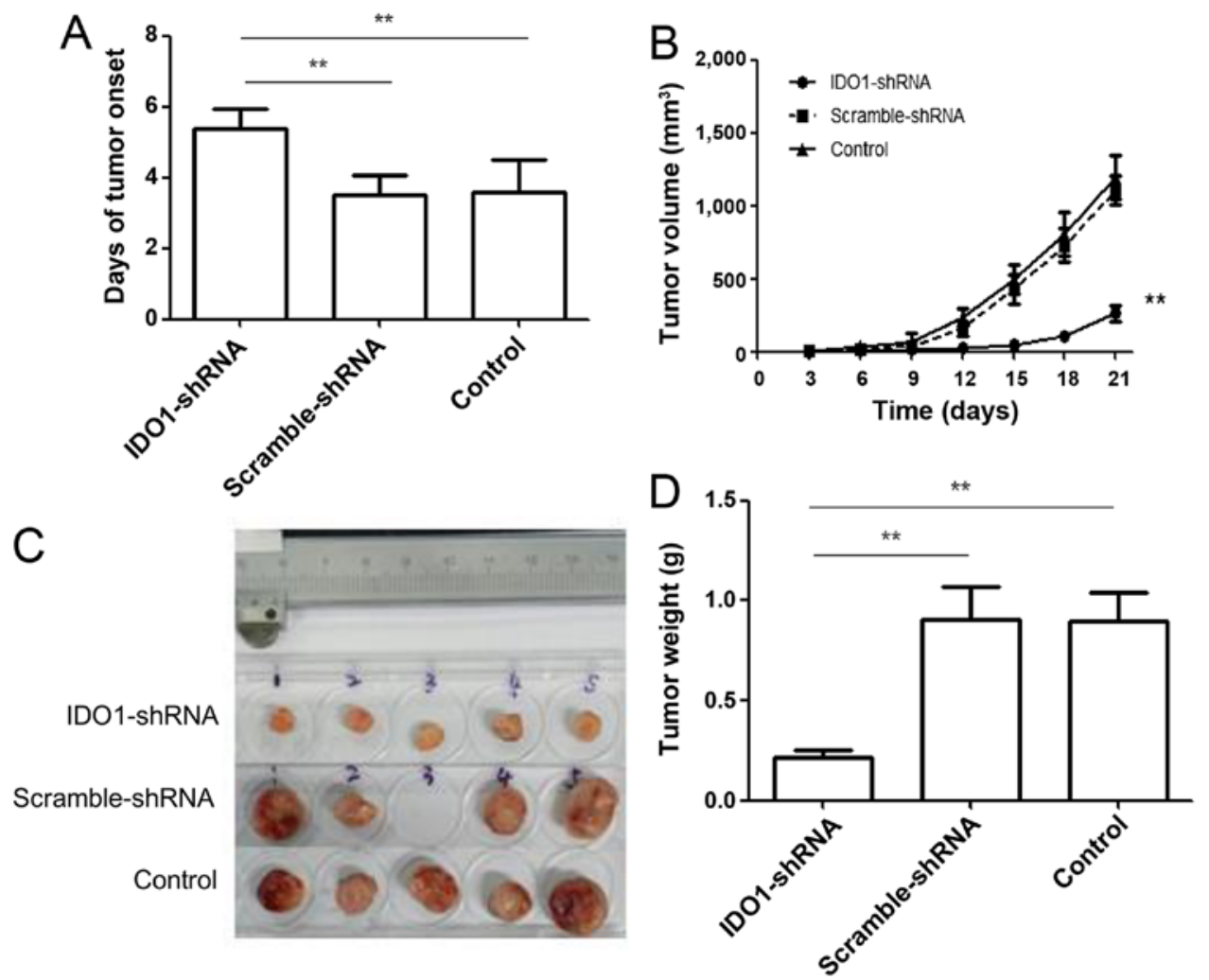

Figure 4. Treatment with IDO1 shRNA in vivo suppresses tumor growth. Lung cancer model was established by inoculating $1 \times 10^{6}$ LLC cells subcutaneously into the upper hind leg of C57BL/6 mice. The cancer-bearing mice were treated with $50 \mu \mathrm{g}$ of IDO1 shRNA or scramble shRNA at days 3, 7, 14 and 21 . (A) Tumor onset day was defined as the time when tumor diameter reached $5 \mathrm{~mm}$. (B) The tumor growth curve (C) and tumor size at day 21. (D) Tumor weight at day 21. Data are expressed as mean $\pm \mathrm{SD}(* * \mathrm{p}<0.01$ vs. scramble shRNA or control groups). 




IDO1-siRNA

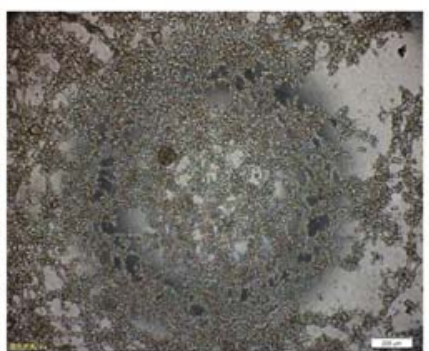

GL2-siRNA

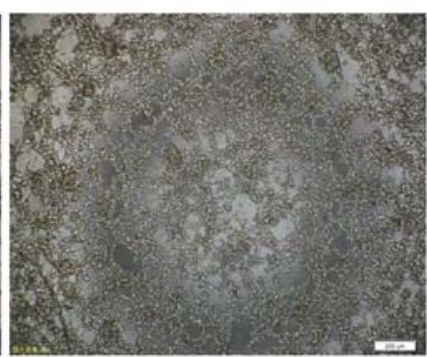

Control

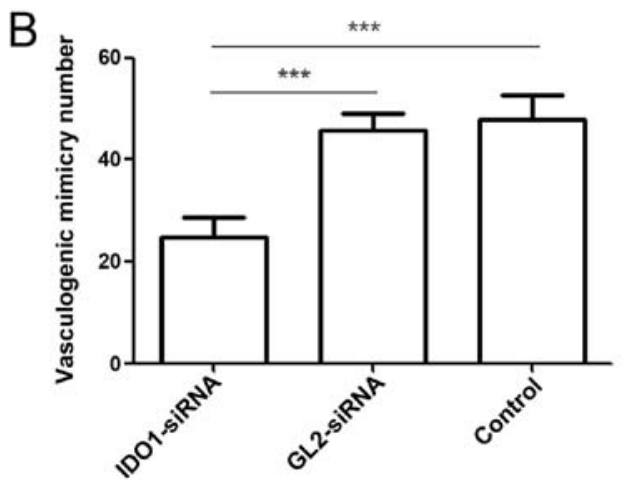

Figure 5. The effect of IDO1 on vasculogenic mimicry (VM) formation of LLC cells. (A) LLC cells were transfected with siRNA targeting IDO1 or GL2 (control siRNA), or remained untransfected as a blank control. At $24 \mathrm{~h}$ after gene silencing, the cells were placed on the Matrigel. After $3 \mathrm{~h}$ culture, formations of VM were observed under a microscope. (B) The number of VM tubers formed by the cells transfected with IDO1 siRNA, GL2 siRNA and control cells were counted. Data are expressed as mean $\pm \operatorname{SD}(* * * 00001)$.
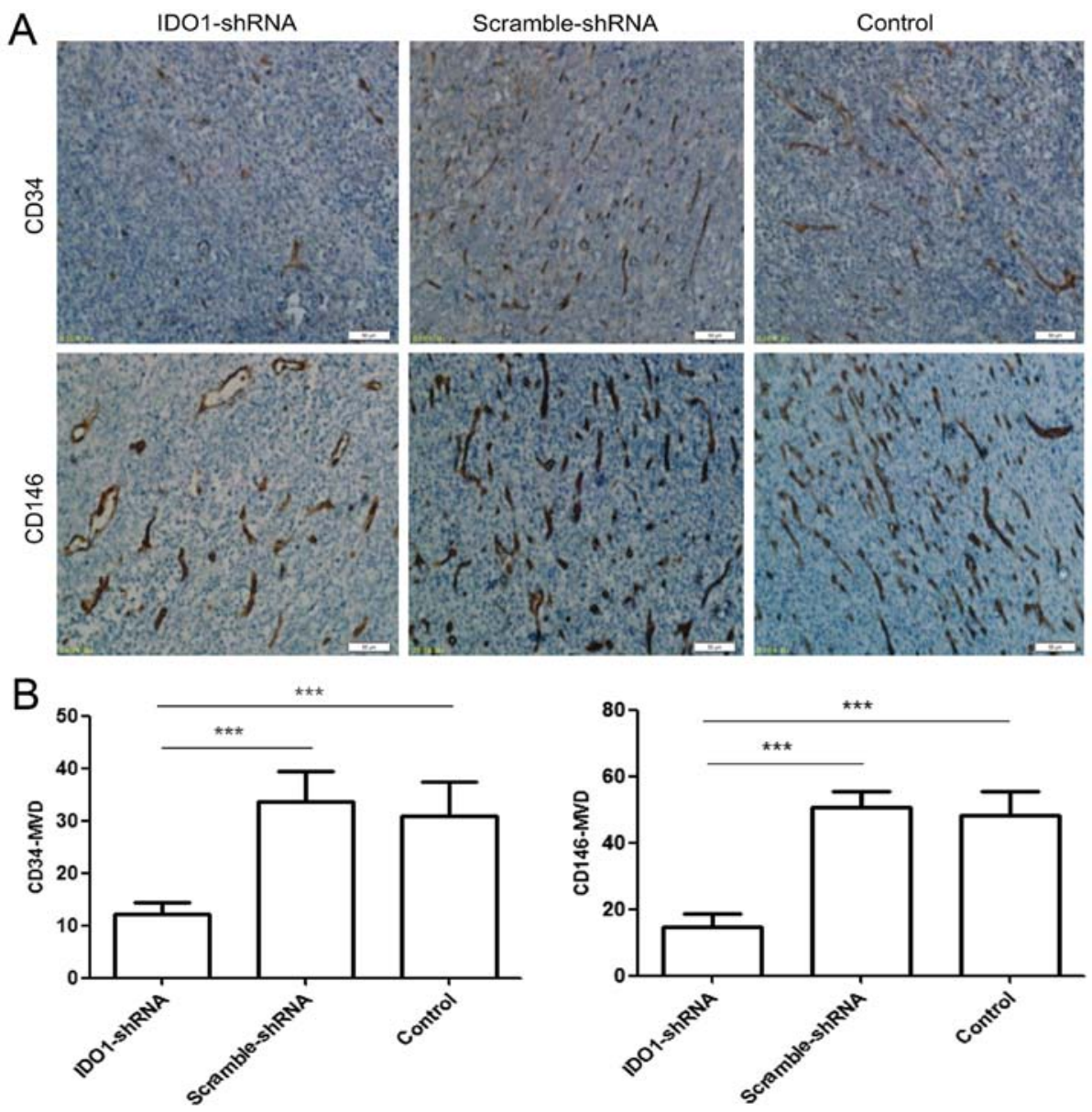

Figure 6. The effect of IDO1 on tumor angiogenesis. (A) Lung cancer-bearing mice were established and treated with IDO1 shRNA or scramble shRNA as described in Fig. 4. Tumor tissues were collected on day 21, and fixed with PFA. The paraffin sections were used for histoimmunochemistry after staining with antibodies against CD34 and CD146, respectively, and observed under a microscope. (B) The number of CD34 ${ }^{+}$MVD and CD146 ${ }^{+}$MVD was counted. Data are expressed as mean $\pm \mathrm{SD}\left({ }^{* * *} \mathrm{p} \leq 0.001\right)$. 


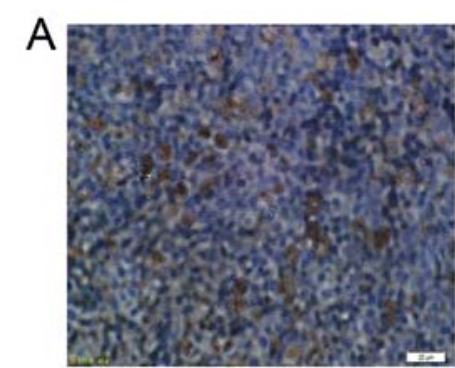

IDO1-shRNA



Scramble-shRNA



Control
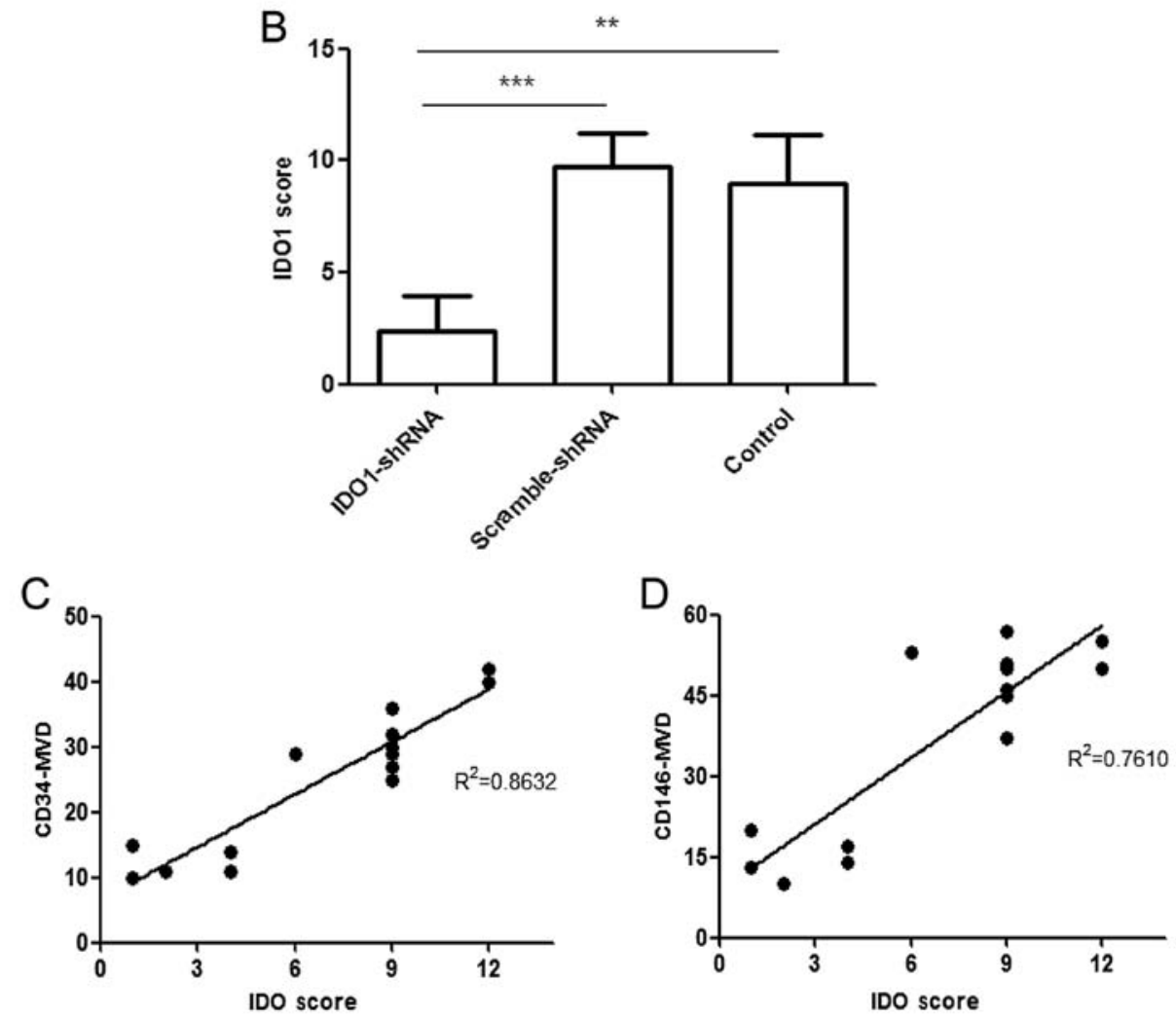

Figure 7. Correlation between MVD and IDO1 expression. (A) In vivo gene silencing of IDO1. Tumor tissues were collected from lung cancer-bearing mice as described in Fig. 6. Expression of IDO1 in the tumors was detected by immunohistochemistry after staining with the antibody specific from IDO1, and observed under a microscope. (B) Scores of IDO1 expression were calculated using staining index as described in Materials and methods. (C) Correlation between IDO1 and CD34 ${ }^{+}$MVD. (D) Correlation between IDO1 and CD146 ${ }^{+}$MVD.

significantly less than $\left(\mathrm{CD} 34^{+} \mathrm{MVD}, 31 \pm 6.442\right.$; CD146 ${ }^{+} \mathrm{MVD}$, $48.4 \pm 7.197)$ those in the control tumors. Thus, IDO1 expression can affect tumor angiogenesis.

Correlation between MVD and IDOI expression. Fig. 7 shows that IDO1 expression was positively correlated to both CD34 ${ }^{+} \mathrm{MVD}\left(\mathrm{r}^{2}=0.8632\right)$ and CD146 ${ }^{+} \mathrm{MVD}\left(\mathrm{r}^{2}=0.761\right)$. This association between the microvascular density marked by CD34 or CD146 and the expression of IDO1 indicates that IDO1 has the potential to participate in the formation of new capillaries.

\section{Discussion}

In this study, we aimed to clarify a factor involved in lung cancer progression, particularly angiogenesis, and to develop molecular therapy that could target it. We focused on IDO1, which has been widely reported to be involved in tumor immunotolerance. First, we attempted to transfect siRNA into the IDO1-expressing Lewis lung cancer (LLC) cell line to knock down the IDO1 gene. qPCR and western blot analysis identified that this gene was silenced successfully. We conducted a basic study using this gene-silenced cell line, and found that the expression of IDO1 can affect the invasion and migration of LLC cells in vitro.

Our research indicates that siRNA or its precursor shRNA can block the expression of the target gene through an RNAi mechanism (24). RNAi is a technique of specific gene silencing at the post-transcriptional level, which can transfect double stranded RNA (dsRNA) of target sequence into an organism. RNAi has the characteristics of high efficiency and gene specificity with the potential to replace gene knockout technology to some extent. Research of RNAi gene silencing in tumor, virus infection, genetic disease and related diseases has entered the stage of clinical trials $(25,26)$. Targeting gene knockout mice using a short hairpin RNA (shRNA) plasmid through hydrodynamic tail vein injection has been reported to treat breast cancer, prostate cancer, liver cancer and other diseases in the mouse model (27-29). In this study, we designed shRNA to silence IDO1 in treating C57BL/6-LLC 
tumor-bearing mice. With hydrodynamic tail vein injection of IDO1 shRNA plasmids, the speed of tumor growth slowed significantly. Tumor volume and weight were also reduced markedly. Immunohistochemistry showed reduced expression of IDO1. The above experimental results indicate that IDO1 can be knocked out by injection of IDO1 shRNA plasmids, which then inhibits tumor angiogenesis and growth.

Maniotis et al (23) reported a new kind of tumor vascularization pattern, named vasculogenic mimicry (VM). The authors found that highly invasive melanoma cells can differentiate into multiple cell phenotypes, which have the characteristics of endothelial cells. This process can lead to de novo vascular structure. A growing number of studies have found that many types of cancer, including breast (30), ovarian (31), prostate (32), and lung (33) contain VM. The high plasticity of tumor cells is the formative basis of VM, which could allow certain malignant cells to obtain an embryonic phenotype. When Maniotis et al (23) discovered VM, they also noted that the metastatic capacity of human melanoma cells can affect the formation of VM. After establishing the LLC cell line in which IDO1 gene was silenced, we used the Matrigel matrix three-dimensional cell culture model to observe the ability of LLC cells to form VM. After knockdown of the IDO1 gene, the number of VM decreased markedly, and this may be associated with the silenced IDO1 gene having a reduced metastatic ability. Therefore, the removal of IDO1 gene leads to a decline in the ability of LLC cells to form VM.

Angiogenesis is an essential process in growth, metastasis and invasion of tumor cells. The interactions among tumor cells, immune regulatory factors, and tumor vessels are inextricably linked in tumor microenvironment. IDO1 is a classical negative immunoregulation factor, but its association with tumor angiogenesis has been rarely studied. As reported by Li et al (34), IDO was believed to promote angiogenesis by tryptophan depletion during skin xenograft. Nonaka et al (35), reported that IDO promotes the peritoneal dissemination of ovarian cancer through angiogenesis. Thus, IDO secreted by tumor cells may facilitate the proliferation, migration, and activiation of endothelial cells, which leads to increased neovascularization. In this study, the levels of CD $34^{+}$MVD and CD146 ${ }^{+}$MVD both decreased significantly after IDO1 shRNA treatment. Enhanced IDO expression was positively correlated with MVD marked by CD34 or CD146. These findings suggest that IDO is involved in lung cancer progression, and the IDO1 gene can be successfully silenced using molecular-targeted therapy.

\section{Acknowledgements}

This study was partially supported by grants from the Natural Science Foundation of China (NSFC, no. 81673009), Jiangxi Science and Technology Innovation Programs (20124ACB00800), Canadian Institute of Health Research (CIHR).

\section{References}

1. Zheng R, Zeng H, Zuo T, Zhang S, Qiao Y, Zhou Q and Chen W: Lung cancer incidence and mortality in China, 2011. Thorac Cancer 7: 94-99, 2016.
2. Rusch VW: Stage III non-small cell lung cancer. Semin Respir Crit Care Med 37: 727-735, 2016.

3. Mellor AL and Munn DH: IDO expression by dendritic cells: Tolerance and tryptophan catabolism. Nat Rev Immunol 4: 762-774, 2004.

4. Yamazaki F, Kuroiwa T, Takikawa O and Kido R: Human indolylamine 2,3-dioxygenase. Its tissue distribution, and characterization of the placental enzyme. Biochem J 230: 635-638, 1985.

5. Grohmann U, Fallarino F and Puccetti P: Tolerance, DCs and tryptophan: Much ado about IDO. Trends Immunol 24: 242-248, 2003.

6. Hirata F, Ohnishi T and Hayaishi O: Indoleamine 2,3-dioxygenase. Characterization and properties of enzyme. O2- complex. J Biol Chem 252: 4637-4642, 1977.

7. Uyttenhove C, Pilotte L, Théate I, Stroobant V, Colau D, Parmentier N, Boon T and Van den Eynde BJ: Evidence for a tumoral immune resistance mechanism based on tryptophan degradation by indoleamine 2,3-dioxygenase. Nat Med 9: 1269-1274, 2003.

8. Karanikas V, Zamanakou M, Kerenidi T, Dahabreh J, Hevas A, Nakou M, Gourgoulianis KI and Germenis AE: Indoleamine 2,3-dioxygenase (IDO) expression in lung cancer. Cancer Biol Ther 6: 1258-1262, 2007.

9. Chen IC, Lee KH, Hsu YH, Wang WR, Chen CM and Cheng YW: Expression pattern and clinicopathological relevance of the indoleamine 2,3-dioxygenase 1/tryptophan 2,3-dioxygenase protein in colorectal cancer. Dis Markers 2016: 8169724, 2016.

10. Feder-Mengus C, Wyler S, Hudolin T, Ruszat R, Bubendorf L, Chiarugi A, Pittelli M, Weber WP, Bachmann A, Gasser TC, et al: High expression of indoleamine 2,3-dioxygenase gene in prostate cancer. Eur J Cancer 44: 2266-2275, 2008.

11. Bi WW, Zhang WH, Yin GH, Luo H, Wang SQ, Wang H, Li C, Yan WQ and Nie DZ: Analysis of indoleamine 2-3 dioxygenase (IDO) and EGFR co-expression in breast cancer tissue by immunohistochemistry. Asian Pac J Cancer Prev 15: 5535-5538, 2014.

12. Liu J, Zhang H, Jia L and Sun H: Effects of Treg cells and IDO on human epithelial ovarian cancer cells under hypoxic conditions. Mol Med Rep 11: 1708-1714, 2015.

13. Creelan BC, Antonia S, Bepler G, Garrett TJ, Simon GR and Soliman HH: Indoleamine 2,3-dioxygenase activity and clinical outcome following induction chemotherapy and concurrent chemoradiation in Stage III non-small cell lung cancer. Oncoimmunology 2: e23428, 2013.

14. Ino K, Yoshida N, Kajiyama H, Shibata K, Yamamoto E, Kidokoro K, Takahashi N, Terauchi M, Nawa A, Nomura S, et al: Indoleamine 2,3-dioxygenase is a novel prognostic indicator for endometrial cancer. Br J Cancer 95: 1555-1561, 2006.

15. Takao M, Okamoto A, Nikaido T, Urashima M, Takakura S, Saito M, Saito M, Okamoto S, Takikawa O, Sasaki H, et al: Increased synthesis of indoleamine-2,3-dioxygenase protein is positively associated with impaired survival in patients with serous-type, but not with other types of, ovarian cancer. Oncol Rep 17: 1333-1339, 2007.

16. Fus LP and Górnicka B: Role of angiogenesis in urothelial bladder carcinoma. Cent European J Urol 69: 258-263, 2016.

17. Cox G, Walker RA, Andi A, Steward WP and O'Byrne KJ: Prognostic significance of platelet and microvessel counts in operable non-small cell lung cancer. Lung Cancer 29: 169-177, 2000.

18. Pereira T, Dodal S, Tamgadge A, Bhalerao S and Tamgadge S: Quantitative evaluation of microvessel density using CD34 in clinical variants of ameloblastoma: An immunohistochemical study. J Oral Maxillofac Pathol 20: 51-58, 2016.

19. Zheng C, Qiu Y, Zeng Q, Zhang Y, Lu D, Yang D, Feng J and Yan X: Endothelial CD146 is required for in vitro tumor-induced angiogenesis: The role of a disulfide bond in signaling and dimerization. Int J Biochem Cell Biol 41: 2163-2172, 2009.

20. Jin EJ, Choi YA, Sonn JK and Kang SS: Suppression of ADAM 10-induced Delta-1 shedding inhibits cell proliferation during the chondro-inhibitory action of TGF-beta3. Mol Cells 24: 139-147, 2007.

21. Scharl A, Vierbuchen M, Conradt B, Moll W, Würz H and Bolte A: Immunohistochemical detection of progesterone receptor in formalin-fixed and paraffin-embedded breast cancer tissue using a monoclonal antibody. Arch Gynecol Obstet 247: 63-71, 1990.

22. Weidner N, Folkman J, Pozza F, Bevilacqua P, Allred EN, Moore DH, Meli S and Gasparini G: Tumor angiogenesis: A new significant and independent prognostic indicator in early-stage breast carcinoma. J Natl Cancer Inst 84: 1875-1887, 1992. 
23. Maniotis AJ, Folberg R, Hess A, Seftor EA, Gardner LM, Pe'er J, Trent JM, Meltzer PS and Hendrix MJ: Vascular channel formation by human melanoma cells in vivo and in vitro: Vasculogenic mimicry. Am J Pathol 155: 739-752, 1999.

24. Jinek M and Doudna JA: A three-dimensional view of the molecular machinery of RNA interference. Nature 457: 405-412, 2009.

25. Lares MR, Rossi JJ and Ouellet DL: RNAi and small interfering RNAs in human disease therapeutic applications. Trends Biotechnol 28: 570-579, 2010.

26. Burnett JC, Rossi JJ and Tiemann K: Current progress of siRNA/ shRNA therapeutics in clinical trials. Biotechnol J 6: 1130-1146, 2011.

27. Zhang X, Liu Y, Zhang G, Shi J, Zhang X, Zheng X, Jiang AT, Zhang ZX, Johnston N, Siu KS, et al: Synergic silencing of costimulatory molecules prevents cardiac allograft rejection. J Transl Med 12: 142, 2014.

28. Vakili S, Ebrahimi SS, Sadeghi A, Gorgani-Firuzjaee S, Beigy M, Pasalar P and Meshkani R: Hydrodynamic-based delivery of PTP1B shRNA reduces plasma glucose levels in diabetic mice. Mol Med Rep 7: 211-216, 2013

29. Caldas H, Holloway MP, Hall BM, Qualman SJ and Altura RA: Survivin-directed RNA interference cocktail is a potent suppressor of tumour growth in vivo. J Med Genet 43: 119-128, 2006.

30. Shirakawa K, Tsuda H, Heike Y, Kato K, Asada R, Inomata M, Sasaki H, Kasumi F, Yoshimoto M, Iwanaga T, et al: Absence of endothelial cells, central necrosis, and fibrosis are associated with aggressive inflammatory breast cancer. Cancer Res 61 445-451, 2001
31. Sood AK, Fletcher MS, Zahn CM, Gruman LM, Coffin JE, Seftor EA and Hendrix MJ: The clinical significance of tumor cell-lined vasculature in ovarian carcinoma: Implications for anti-vasculogenic therapy. Cancer Biol Ther 1: 661-664, 2002.

32. Sharma N, Seftor RE, Seftor EA, Gruman LM, Heidger PM Jr, Cohen MB, Lubaroff DM and Hendrix MJ: Prostatic tumor cell plasticity involves cooperative interactions of distinct phenotypic subpopulations: Role in vasculogenic mimicry. Prostate 50: 189-201, 2002.

33. Passalidou E, Trivella M, Singh N, Ferguson M, Hu J, Cesario A, Granone P, Nicholson AG, Goldstraw P, Ratcliffe C, et al: Vascular phenotype in angiogenic and non-angiogenic lung non-small cell carcinomas. Br J Cancer 86: 244-249, 2002.

34. Li Y, Tredget EE, Ghaffari A, Lin X, Kilani RT and Ghahary A: Local expression of indoleamine 2,3-dioxygenase protects engraftment of xenogeneic skin substitute. J Invest Dermatol 126: 128-136, 2006.

35. Nonaka H, Saga Y, Fujiwara H, Akimoto H, Yamada A, Kagawa S, Takei Y, Machida S, Takikawa O and Suzuki M: Indoleamine 2,3-dioxygenase promotes peritoneal dissemination of ovarian cancer through inhibition of natural killer cell function and angiogenesis promotion. Int J Oncol 38: 113-120, 2011. 